\title{
An omega result for supremum norms of Hecke-eigenforms in the level aspect
}

\author{
Yuk-Kam Lau \\ Department of Mathematics, The University of Hong Kong, Pokfulam Road, Hong Kong \\ (email: yklau@maths.hku.hk)
}

\begin{abstract}
This note is to remark the large supremum norms of some Hecke-eigenforms of large squarefree levels and nebentypus of small conductors, based on the recent prominent progress by Soundararajan on the extreme central values of $L$-functions.
\end{abstract}

Keywords: Hecke-eigenforms, Central values of $L$-functions

$\operatorname{MSC}(2000): \quad 11 F 12$

\section{Introduction}

Arithmetic quantum chaos refer to the investigation of quantum chaos endowed with arithmetic structures; in physical terms, it is concerned with the study of the semi-classical properties of quantum systems whose classical limit is a chaotic Hamiltonian system. There are many interesting problems in this subject, which are often linked to objects and questions in number theory (due to the arithmetic nature). Interested readers may pursue the articles [20], [4], [16], etc. How the individual eigenstate localizes is one of the central topics. Under the condition of ergodic geodesic flows, almost all eigenstates become equidistributed, but on the other hand, scarrings are observed in various numerical experiments. Motivated by their result of no strong scarring on closed geodesics, Rudnick and Sarnak [19] proposed the conjecture of quantum unique ergodicity (QUE). This challenging problem and the analogue for holomorphic Hecke eigenforms are just settled ingeniously in Soundararajan [24] and Holowinsky \& Soundararajan [8]. (See [22] and the webpage of AIM at "http://www.aimath.org/news/que/" for more information and the development of the breakthrough.) In addition to equidistributions, these works constitute an incentive to another vital topic - the norms of the eigenstates.

Let $\Gamma=S L(2, \mathbb{Z})$ be the full modular group, and $\mathbb{H}$ be the upper half plane. Then $\Gamma \backslash \mathbb{H}$ is a Riemannian manifold whose metric is $y^{-2}\left(d x^{2}+d y^{2}\right)$. The eigenstates, which are the eigenfunctions of the Laplacian $\Delta$, are commonly known as the Maass (cusp) forms. The Hecke-Maass forms are those forms which are simultaneously the eigenfunctions of all Hecke

The work described in this paper was fully supported by a grant from the Research Grants Council of the Hong Kong Special Administrative Region, China (HKU 7032/06P). 
operators. For a Hecke-Maass form $\varphi_{j}$, it is shown (see [21, p.38-40]) that

$$
t_{j}^{1 / 6-\varepsilon}\left\|\varphi_{j}\right\|_{2} \ll\left\|\varphi_{j}\right\|_{\infty} \ll t_{j}^{1 / 6+\varepsilon}\left\|\varphi_{j}\right\|_{2}
$$

where $1 / 4+t_{j}^{2}$ is the eigenvalue of $\Delta$ for $\varphi_{j}$, and $\|\cdot\|_{\infty}$ and $\|\cdot\|_{2}$ are respectively the supremum and $L^{2}$ norms. An analogous result holds true for the holomorphic forms. Holomorphic cusp forms of (even) weight $k$ for $\Gamma$ descend to functions on $\Gamma \backslash \mathbb{H}$. Under the realization $\mathbb{H}=$ $S L(2, \mathbb{R}) / S O(2, \mathbb{R})$, they are lifted to the unit cotangent bundle to $\Gamma \backslash \mathbb{H}$ and form eigenfunctions of a Laplacian $\widetilde{\Delta}$ with the eigenvalue $k(k-2) / 4$. A holomorphic cusp form $f$ is primitive if it is a common eigenfunction of all Hecke operators with the normalization $T_{1} f=f$. In [25], Xia proved that for any primitive form $f$ of weight $k$ for $\Gamma=S L(2, \mathbb{Z})$,

$$
k^{1 / 4-\varepsilon}\left\|F_{f}\right\|_{2} \ll\left\|F_{f}\right\|_{\infty} \ll k^{1 / 4+\varepsilon}\left\|F_{f}\right\|_{2}
$$

where $F(z)=y^{k / 2} f(z)$.

At the first sight, the large supremum norms in (1.1) and (1.2) may not be expected in light of the equidistribution property from QUE. Interestingly some large values are found in the high horocycles. Let us illustrate with the holomorphc forms for the full modular group. A primitive form of weight $k$ admits the Fourier series expansion

$$
f(z)=\sum_{n \geq 1} \lambda_{f}(n) n^{(k-1) / 2} e(n z)
$$

where $e(x)=\exp (2 \pi i x)$. Integrating over the horocycle $\Im m z=y$, we have for $y \leq k$,

$$
\int_{0}^{1} y^{k}|f(x+i y)|^{2} d x=y \sum_{n \geq 1}\left|\lambda_{f}(n)\right|^{2}(n y)^{k-1} e^{-4 \pi n y} .
$$

Clearly it is $\geq y^{k} e^{-4 \pi y}$. Applying the vital inequality of Deligne $\left|\lambda_{f}(n)\right| \leq d(n) \ll n^{\varepsilon}$, we can give an upper bound via the observation that $\xi^{k-1+\varepsilon} e^{-4 \pi \xi}$ is increaing on $\left(0, \xi_{0}\right)$ and decreasing on $\left(\xi_{0}, \infty\right)$, where $\xi_{0}=(k-1+\varepsilon) /(4 \pi)$. Hence it is also

$$
\ll \frac{y}{k}\left(\frac{k}{4 \pi e}\right)^{k+\varepsilon}+\int_{0}^{\infty} t^{k-1+\varepsilon} e^{-4 \pi t} d t .
$$

Since the $L^{2}$-norm of $f$ is

$$
\|f\|_{2}^{2}=\frac{2}{\pi} \frac{\Gamma(k)}{(4 \pi)^{k}} L\left(1, \operatorname{sym}^{2} f\right)=k^{-1 / 2+o(1)}\left(\frac{k}{4 \pi e}\right)^{k}
$$

by [9] and [10], we deduce that

$$
\|f\|_{2}^{2} \frac{y}{\sqrt{k}}\left(\frac{4 \pi y}{k}\right)^{k} e^{k-4 \pi y} k^{-\varepsilon} \ll \int_{0}^{1} y^{k}|f(x+i y)|^{2} d x \ll\|f\|_{2}^{2}\left(1+\frac{y}{\sqrt{k}}\right)\left(\frac{k}{y}\right)^{\varepsilon} .
$$

The lower bound in (1.2) follows by taking $y=k /(4 \pi)$, and it also alludes heuristically that the size of $f$ is small over low horocycles.

In addition to the eigenvalues, it was investigated the supremum norms in the level aspect. Let $\Gamma=\Gamma_{0}(N)$ be a congruence subgroup where $N$ is squarefree. Let $\left\{f_{1}, \cdots, f_{g}\right\}$ be the set 
Omega result for supremum norms of Hecke-eigenforms

of all primitive forms of weight 2 for $\Gamma$. In [1] and [17], it was derived very good estimates for

$$
\sup _{z \in \mathbb{H}} \sum_{j=1}^{g} \frac{(\Im m z)^{2}\left|f_{j}(z)\right|^{2}}{\left\|f_{j}\right\|^{2}}
$$

where

$$
\|f\|^{2}=\int_{\Gamma \backslash \mathbb{H}} y^{2}|f(z)|^{2} \frac{d x d y}{y^{2}} .
$$

Later Jorgenson and Kramer [15] investigated a more general context and obtained the result

$$
\sup _{z \in \mathbb{H}} \sum_{j=1}^{g}(\Im m z)^{2}\left|f_{j}(z)\right|^{2} /\left\|f_{j}\right\|^{2} \ll 1
$$

which is the best possible in order of magnitude. For individual primitive forms of weight $k$ and level $N$ with nebentypus, Blomer and Holowinsky [3] recently proved the interesting nontrivial result

$$
\left\|F_{f}\right\|_{\infty} \ll N^{-\delta}\left\|F_{f}\right\|_{2}
$$

with $\delta=1 / 38$ if $f$ is primitive. As in above, we do not normalize the volume measure, and take

$$
\|f\|_{2}^{2}=\left\|F_{f}\right\|_{2}^{2}=\int_{\Gamma \backslash \mathbb{H}} y^{k}|f(z)|^{2} \frac{d x d y}{y^{2}} .
$$

Applying the argument for (1.3), it is easily shown that

$$
\left\|F_{f}\right\|_{\infty} \gg N^{-1 / 2-\varepsilon}\left\|F_{f}\right\|_{2}
$$

By virtue of (1.4) (in which $g$ is known to be about $N$ ), the true value of $\delta$ in (1.5) is anticipated to be $1 / 2$ up to $o(1)$ and the lower estimate in (1.6) may be quite sharp. So it could be delicate but is desired to find primitive forms with bigger supremum norms. Our purpose is to illustrate their existence.

Theorem 1. Let $N \geq 1$ be squarefree, $k \geq 2$ an even integer and $\chi$ be a character mod $N$ of conductor $N^{*}$ with $\chi(-1)=(-1)^{k}$. Assume one of the following condition holds for the Dirichlet $L$-function $L(s, \chi)$.

(1) $L(s, \chi)$ has no Siegel zero and $N^{*} \leq \exp \left(c \sqrt{\log _{2} N}\right)$ for some absolute constant $c>0$.

(2) $L(s, \chi)$ has a Siegel zero and $N^{*} \leq c(\varepsilon)\left(\log _{2} N\right)^{1 / \varepsilon}$ for some ineffective positive constant $c(\varepsilon)$ and for any $\varepsilon>0$.

(3) The Grand Riemann Hypothesis for $L(s, \chi)$ is valid (and $N^{*} \leq N$ ).

There exists a primitive form $f$ of weight $k$ with nebentypus $\chi$ for the congruence subgroup $\Gamma_{0}(N)$ such that

$$
\frac{\sup _{z \in \mathbb{H}} y^{k / 2}|f(z)|}{\sqrt{\langle f, f\rangle}} \geq N^{-1 / 2} \exp \left(\left(\frac{1}{2}+o(1)\right) \sqrt{\frac{\log N}{\log _{2} N}}\right)
$$


where $\log _{r}$ is the rth iterated logarithm, so $\log _{2}=\log \log$, and

$$
\langle f, g\rangle=\int_{\Gamma_{0}(N) \backslash \mathbb{H}} y^{k} f(z) \overline{g(z)} \frac{d x d y}{y^{2}}
$$

denotes the Petersson inner product.

The method is simply to pass the problem to the central values of $L$-functions, and then apply the robust "resonance" method, newly developed by Soundararajan [23]. Indeed Theorem 1 follows from the result below. Both will be proved in Section 3.

Theorem 2. Under the same conditions as in Theorem 1, there exists a primitive form $f$ of even weight $k$ for $\Gamma_{0}(N)$ with nebentypus $\chi$ whose conductor satisfies one of (1)-(3) in Theorem 1 such that

$$
|L(1 / 2, f)| \geq \exp \left(\left(\frac{1}{2}+o(1)\right) \sqrt{\frac{\log N}{\log _{2} N}}\right) .
$$

We end this section with some remarks.

Remark 1. The case for trivial nebentypus character corresponds to $N^{*}=1$, and $\chi=\psi_{N}$ (and $N$ is still assumed to be squarefree). Theorems $1 \& 2$ hence hold valid.

Remark 2. The conditions on $L(s, \chi)$ are imposed to suppress the character sum in (3.25). To bypass the brute force treatment, a natural remedy is to select skillfully the factors for resonance. In view of (3.23), it is tempted to align $\chi(p)$ by adding a rotating factor to $r(p)$. More concretely, suppose $\chi(p)=e^{i \theta}$ and $r(p)=|r(p)| e^{-\phi}$, then the factor $\Re e(\overline{r(p)}+\chi(p) r(p))$ equals

$$
2|r(p)| \cos \frac{\theta}{2} \cos \left(\frac{\theta}{2}-\phi\right)
$$

which can, however, be very small, i.e. $\theta \approx \pi$ or $\chi(p) \approx-1$. This case perhaps cannot occur simultaneously for all $p$ where $r(\cdot)$ is supported. But we have no method to take any advantage. It is also very likely to relax the size of the conductor $N^{*}$ in conditions (1) or (2) if we allow some exceptional nebentypus.

\section{Preliminaries}

Let $N \geq 1$ be squarefree, $k \geq 2$ an even integer and $\chi$ be a Dirichlet character mod $N$ induced by a primitive character $\chi^{*} \bmod N^{*}$ where $N=N^{\prime} N^{*}$. Thus, $\chi=\psi_{N^{\prime}} \chi^{*}$ where $\psi_{N^{\prime}}$ is the trivial character mod $N^{\prime}$. Assume $\chi(-1)=(-1)^{k}$ and write $\Gamma=\Gamma_{0}(N)$. Define the slash operator for $A \in G L_{2}^{+}(\mathbb{R})$ by

$$
\left.f\right|_{A}(z):=(\operatorname{det} A)^{k / 2} j(A, z)^{-k} f(A z) .
$$

where

$$
j(A, z)=c z+d \quad \text { if } A=\left(\begin{array}{ll}
a & b \\
c & d
\end{array}\right) .
$$


Omega result for supremum norms of Hecke-eigenforms

A holomorphic cusp form of weight $k$ and nebentypus $\chi$ for $\Gamma$ is a holomorphic function $f$ : $\mathbb{H} \rightarrow \mathbb{C}$ that satisfies $\left.f\right|_{\gamma}(z)=\chi(d) f(z)$, or equivalently

$$
f\left(\frac{a z+b}{c z+d}\right)=\chi(d)(c z+d)^{k} f(z), \quad \text { for every } \gamma=\left(\begin{array}{ll}
a & b \\
c & d
\end{array}\right) \in \Gamma
$$

and decays rapidly at each cusp. The collection of these forms yield a finite dimensional vector space $S_{k}(N, \chi)$. By primitive forms, we mean the normalized elements $f \in S_{k}^{*}(N, \chi)$ (the space of newforms, see [2] or $[13, \S 14.7])$ which are simultaneously the eigenfunctions of all Hecke operators $T_{n}$ and the involution operator $\bar{W}$, i.e.

$$
\bar{W} f(z):=(-1)^{k} N^{-k / 2} z^{-k} \overline{f(1 /(N \bar{z}))}=\eta_{f} f(z)
$$

for some $\eta_{f} \in \mathbb{C}$. The normalization is taken such that the the Fourier expansion of $f$ at the cusp $\infty$ is

$$
f(z)=\sum_{n \geq 1} \lambda_{f}(n) n^{(k-1) / 2} e(n z)
$$

where $e(x):=e^{2 \pi i x}$ and $\lambda_{f}(n) n^{(k-1) / 2}$ is the eigenvalue of $T_{n}$. We have $\lambda_{f}(1)=1$,

$$
\begin{cases}\left|\lambda_{f}(p)\right|=1 & \text { if } p \mid N^{*}, \\ \lambda_{f}(p)^{2}=\chi^{*}(p) p^{-1} & \text { if } p \mid N^{\prime} \text { and } \\ \lambda_{f}(m) \lambda_{f}(n)=\sum_{d \mid(m, n)} \chi(d) \lambda_{f}\left(\frac{m n}{d^{2}}\right) & \text { for all } m, n \geq 1\end{cases}
$$

from [14, Theorem 3] by setting $a_{f}(n)=\lambda_{f}(n) n^{(k-1) / 2}$ therein. In particular, $\lambda_{f}(m n)=$ $\lambda_{f}(m) \lambda_{f}(n)$ if $n \mid N^{\infty}$. Moreover, it follows from [5] that $\left|\lambda_{f}(p)\right| \leq 2$ for any prime $p \nmid N$, whence there are $\alpha_{p}, \beta_{p} \in \mathbb{C}$ with $\left|\alpha_{p}\right|=\left|\beta_{p}\right|=1$ for which

$$
\lambda_{f}(p)=\alpha_{p}+\beta_{p} \quad \text { and } \quad \chi(p)=\alpha_{p} \beta_{p} \quad(p \nmid N) .
$$

We thus deduce that

$$
\lambda_{f}\left(p^{r}\right)=\frac{\alpha_{p}^{r+1}-\beta_{p}^{r+1}}{\alpha_{p}-\beta_{p}} \quad(p \nmid N)
$$

and $\overline{\lambda_{f}(n)}=\overline{\chi(n)} \lambda_{f}(n)$ for $(n, N)=1$.

The eigenvalue $\eta_{f}$ of $\bar{W}$ satisfies $\left|\eta_{f}\right|=1$ as $\bar{W}$ is an involution, and

$$
\mu\left(N^{\prime}\right) \overline{\chi^{*}\left(N^{\prime}\right)} \tau\left(\overline{\chi^{*}}\right)=\tau(\bar{\chi})=\eta_{f} \overline{\lambda_{f}(N)} \sqrt{N}
$$

This can be seen through the proof of [11, Theorem 6.29], as follows. Let $f \in S_{k}(N, \chi)$ be a primitive form. Using the identity

$$
\left(\begin{array}{cc}
1 & u / N \\
& 1
\end{array}\right)\left(\begin{array}{cc} 
& -1 / N \\
N &
\end{array}\right)\left(\begin{array}{cc}
1 & v / N \\
& 1
\end{array}\right)=\left(\begin{array}{cc}
u & (u v-1) / N \\
N & v
\end{array}\right),
$$

we have

$$
f\left|\left(\begin{array}{cc}
1 & u / N \\
& 1
\end{array}\right)\left(\begin{array}{ll} 
& -1 / N \\
N &
\end{array}\right)=\chi(v) f\right|\left(\begin{array}{cc}
1 & -v / N \\
& 1
\end{array}\right)
$$


where $v$ is chosen to fulfil $u v \equiv 1(\bmod N)$. Define

$$
\left.g:=\left.\sum_{u(\bmod N)}^{*} f\right|^{1} \begin{array}{cc}
u / N \\
1
\end{array}\right)\left(\begin{array}{ll} 
& -1 / N \\
N &
\end{array}\right)=\sum_{v(\bmod N)} \chi(v) f \mid\left(\begin{array}{cc}
1 & -v / N \\
1
\end{array}\right)
$$

and

$$
K g(z)=\overline{g(-\bar{z})}
$$

Then (2.4) follows from the first coefficient of $K g$. From the second equality in (2.5),

$$
g(z)=\sum_{v(\bmod N)} \chi(v) f\left(z-\frac{v}{N}\right)=\sum_{n=1}^{\infty} b(n) n^{(k-1) / 2} e(n z)
$$

where

$$
b(n)=\lambda_{f}(n) \sum_{v(\bmod N)} \chi(v) e(-n v / N) .
$$

By [6, Chapter 9], we have for $(n, N)=1$,

$$
b(n)=\overline{\tau(\bar{\chi}) \chi(n)} \lambda_{f}(n)
$$

In case $\chi$ is primitive, it holds even for $(n, N)>1$ by [6]. Otherwise, we may derive the formula

$$
b(n)=\lambda_{f}(n) \overline{\tau(\bar{\chi}) \chi^{*}(n)} \sum_{d \mid\left(n, N^{\prime}\right)} d \mu(d) .
$$

The proof goes along the argument in [6, p.67], where it yields immediately

$$
\tau(\bar{\chi})=\mu\left(N^{\prime}\right) \overline{\chi^{*}\left(N^{\prime}\right)} \tau\left(\overline{\chi^{*}}\right) .
$$

Putting $\chi=\psi_{N} \chi^{*}$ and relaxing the condition $(u, N)=1$ by Moebius formula, we may express

$$
\begin{aligned}
& \sum_{v(\bmod N)} \chi(v) e(-n v / N) \\
= & \sum_{d \mid N} \mu(d) \sum_{v(\bmod N / d)} \chi^{*}(d v) e\left(-\frac{n v}{N / d}\right) \\
= & \sum_{d \mid N^{\prime}} \mu(d) \chi^{*}(d) \sum_{t\left(\bmod N^{*}\right)} \chi^{*}(t) e\left(-\frac{n t}{N / d}\right) \sum_{l\left(\bmod N^{\prime} / d\right)} e\left(-\frac{n l}{N^{\prime} / d}\right)
\end{aligned}
$$

with the substitution $v=l N^{*}+t$. Therefore, this reduces to

$$
\begin{aligned}
& N^{\prime} \overline{\tau\left(\overline{\left.\chi^{*}\right)}\right.} \sum_{d\left|N^{\prime},\left(N^{\prime} / d\right)\right| n} \frac{\mu(d) \chi^{*}(d)}{d} \overline{\chi^{*}\left(\frac{n}{N^{\prime} / d}\right)}
\end{aligned}
$$

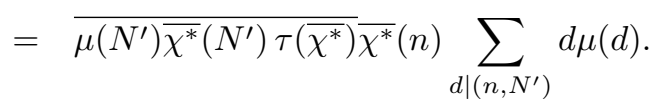

Now (2.9) follows by (2.8) and (2.10). 
Omega result for supremum norms of Hecke-eigenforms

By (2.6) and (2.7),

$$
K g(z)=\sum_{n \geq 1} \overline{b(n)} n^{(k-1) / 2} e(n z)
$$

Next we evaluate with the first equality of (2.5). Relaxing the condition $(u, N)=1$, we see that

$$
\begin{aligned}
& \left.\sum_{u(\bmod N)}^{*} f\right|_{\left(\begin{array}{cc}
1 & u / N \\
1
\end{array}\right)}(z)=\sum_{a d=N} \mu(a) d \sum_{\substack{n \geq 1 \\
d \mid n}} \lambda_{f}(n) n^{(k-1) / 2} e(n z) \\
& \left.=\left.\sum_{a d=N} \mu(a) d^{1 / 2} \lambda_{f}(d) f\right|_{(} \begin{array}{ll}
d & \\
& 1
\end{array}\right),
\end{aligned}
$$

noting that $d \mid N \Rightarrow \lambda_{f}(m d)=\lambda_{f}(m) \lambda_{f}(d)$. As

$$
\left(\begin{array}{ll}
d & \\
& 1
\end{array}\right)\left(\begin{array}{ll}
N^{-1 / N} & -1
\end{array}\right)=\left(\begin{array}{ll} 
& -1 \\
N &
\end{array}\right)\left(\begin{array}{ll}
1 & \\
& 1 / a
\end{array}\right)
$$

for $N=a d$, we have

$$
g=\sum_{a d=N} \mu(a) d^{1 / 2} \lambda_{f}(d) f \mid\left(\begin{array}{ll} 
& -1 \\
N &
\end{array}\right)\left(\begin{array}{ll}
1 & \\
& 1 / a
\end{array}\right) .
$$

Observe from (2.6) and (2.1) that

$$
K\left(\left.f\right|_{A}\right)=\left.(K f)\right|_{A} \quad \text { for } A=\left(\begin{array}{ll}
\alpha & \\
& \delta
\end{array}\right) \in G L_{2}^{+}(\mathbb{R}),
$$

and

$$
K\left(f \mid\left(\begin{array}{ll} 
& -1 \\
N &
\end{array}\right)=\bar{W} f\right.
$$

We deduce from (2.12) that

$$
\left.K g=\eta_{f} \sum_{a d=N} \mu(a) d^{1 / 2} \overline{\lambda_{f}(d)} f \mid \begin{array}{ll}
1 & \\
& 1 / a
\end{array}\right),
$$

and hence conclude with (2.11),

$$
\begin{aligned}
& \sum_{n \geq 1} \overline{b(n)} n^{(k-1) / 2} e(n z) \\
= & \eta_{f} N^{1 / 2} \sum_{n \geq 1} \sum_{a d=N} \mu(a) \overline{\lambda_{f}(d)} \lambda_{f}(n)(a n)^{(k-1) / 2} e(a n z) .
\end{aligned}
$$

We get our result by comparing the coefficient of $e(z)$ with (2.9). Indeed, the equation (2.13) yields the various properties of $\lambda_{f}(p)$. From (2.9), we have

$$
\overline{b(p)}= \begin{cases}\tau(\bar{\chi}) \chi^{*}(p) \overline{\lambda_{f}(p)} & \text { if } p \nmid N, \\ \tau(\bar{\chi}) \chi^{*}(p)(1-p) \overline{\lambda_{f}(p)} & \text { if } p \mid N^{\prime} \\ 0 & \text { if } p \mid N^{*}\end{cases}
$$


The coefficient of $e(p z)$ in $(2.13)$ is

$$
p^{(k-1) / 2} \times \begin{cases}\eta_{f} \sqrt{N} \overline{\lambda_{f}(N)} \lambda_{f}(p) & \text { if } p \nmid N, \\ \eta_{f} \sqrt{N} \overline{\lambda_{f}(N / p)}\left(\left|\lambda_{f}(p)\right|^{2}-1\right) & \text { if } p \mid N .\end{cases}
$$

By virtue of $(2.4)$, we see immediately that $\lambda_{f}(p)=\chi^{*}(p) \overline{\lambda_{f}(p)}$ for $p \nmid N$ and $\left|\lambda_{f}(p)\right|^{2}=1$ for $p \mid N^{*}$. Moreover, for $p \mid N^{\prime}$ we obtain

$$
\chi^{*}(p)(1-p)\left(\overline{\lambda_{f}(p)}\right)^{2}=\left|\lambda_{f}(p)\right|^{2}-1 .
$$

This implies $(p-1)\left|\lambda_{f}(p)\right|^{2}=\left.|| \lambda_{f}(p)\right|^{2}-1 \mid$, i.e.

$$
(p-1)\left|\lambda_{f}(p)\right|^{2}= \pm\left(\left|\lambda_{f}(p)\right|^{2}-1\right) .
$$

Only the "-" case is possible, for $(p-2)\left|\lambda_{f}(p)\right|^{2}$ is nonnegative. Thus $\left|\lambda_{f}(p)\right|^{2}=p^{-1}$ and by (2.14), $\lambda_{f}(p)^{2}=\chi^{*}(p) p^{-1}$.

Now we follow closely the investigation in [12] to derive tools for later use. Let $\mathrm{H}_{k}^{*}\left(M, \chi^{*}\right)$ be the set of all primitive forms in $S_{k}\left(M N^{*}, \psi_{M} \chi^{*}\right)$. By the theory of newforms and oldforms (see [18], [2], [14]), we have the orthogonal decomposition

$$
S_{k}(N, \chi)=\bigoplus_{L M=N^{\prime}} \bigoplus_{f \in \mathrm{H}_{k}^{*}\left(M, \chi^{*}\right)} \operatorname{Span}_{\mathbb{C}}\left\{f_{\mid l}: l \mid L\right\} \quad \text { (orthogonally), }
$$

where $f_{\mid l}(z):=l^{k / 2} f(l z)$ and $\operatorname{Span}_{\mathbb{C}} S$ denotes the subspace generated by elements in $S$ over $\mathbb{C}$.

Let $M \mid N^{\prime}$ and $f \in S_{k}\left(M N^{*}, \psi_{M} \chi^{*}\right)$. Then $f$ also lies in

$$
S_{k}(N, \chi)=S_{k}\left(N^{\prime} N^{*}, \psi_{N^{\prime}} \chi^{*}\right) .
$$

The Petersson inner products in the two spaces differ by a scalar factor. More concretely, let us denote the inner product in $S_{k}\left(M N^{*}, \psi_{M} \chi^{*}\right)$ by

$$
(f, g)_{M}=\int_{\Gamma_{0}\left(M N^{*}\right) \backslash \mathbb{H}} y^{k} f(z) \overline{g(z)} \frac{d x d y}{y^{2}} .
$$

Then, for $f, g \in S_{k}\left(M N^{*}, \psi_{M} \chi^{*}\right)$ we have

$$
(f, g)_{N^{\prime}}=\frac{\nu\left(N^{\prime}\right)}{\nu(M)}(f, g)_{M}
$$

where $\nu(M):=M \prod_{p \mid M}\left(1+p^{-1}\right)$. Note that $\nu(N)=\left[\Gamma_{0}(1): \Gamma_{0}(N)\right]$. Now we investigate a trace formula for primitive forms, following the same analysis in [12, Section 2].

Lemma 2.1. Let $L M=N^{\prime}$ (squarefree). If $f \in \mathrm{H}_{k}^{*}\left(M, \chi^{*}\right)$ and $l_{i} \mid L(i=1,2)$, then with the notation in (2.16), we have

$$
\left(f_{\mid l_{1}}, f_{\mid l_{2}}\right)_{N^{\prime}}=\frac{\overline{\lambda_{f}\left(\ell_{1}\right)} \sqrt{\ell_{1}}}{\nu\left(\ell_{1}\right)} \frac{\lambda_{f}\left(\ell_{2}\right) \sqrt{\ell_{2}}}{\nu\left(\ell_{2}\right)}(f, f)_{N^{\prime}}
$$

where $\ell_{i}=l_{i} /\left(l_{1}, l_{2}\right)$ and $\left(l_{1}, l_{2}\right)$ is the greatest common divisor of $l_{1}$ and $l_{2}$. 
Omega result for supremum norms of Hecke-eigenforms

Proof. Let $F(s)=\left(E(z, s) f\left(l_{1} z\right), f\left(l_{2} z\right)\right)_{N^{\prime}}$ where $E(z, s)=\sum_{\gamma \in \Gamma_{\infty} \backslash \Gamma_{0}(N)}(\Im m \gamma z)^{s}$ and $N=N^{\prime} N^{*}$. By the unfolding method, we get that

$$
\begin{aligned}
F(s) & =\int_{0}^{\infty} y^{s+k-2} \int_{0}^{1} f\left(l_{1} z\right) \overline{f\left(l_{2} z\right)} d x d y \\
& =\frac{\Gamma(s+k-1)}{(4 \pi)^{s+k-1}} \sum_{l_{1} n_{1}=l_{2} n_{2}} \lambda_{f}\left(n_{1}\right) \overline{\lambda_{f}\left(n_{2}\right)}\left(n_{1} n_{2}\right)^{(k-1) / 2}\left(l_{1} n_{1}\right)^{1-k-s} .
\end{aligned}
$$

Writing $\ell_{i}=l_{i} /\left(l_{1}, l_{2}\right)$, then we have

$$
\left(l_{1} l_{2}\right)^{k / 2} F(s)=\frac{\Gamma(s+k-1)}{(4 \pi)^{s+k-1}\left(l_{1}, l_{2}\right)^{s-1}}\left(\ell_{1} \ell_{2}\right)^{1 / 2-s} \sum_{n \geq 1} \lambda_{f}\left(\ell_{1} n\right) \overline{\lambda_{f}\left(\ell_{2} n\right)} n^{-s} .
$$

As $\left(\ell_{1}, \ell_{2}\right)=1$ and $\ell_{1}, \ell_{2}$ are squarefree, the Dirichlet series factors into

$$
\sum_{n \geq 1} \lambda_{f}\left(\ell_{1} n\right) \overline{\lambda_{f}\left(\ell_{2} n\right)} n^{-s}=\prod_{1} \prod_{2} \sum_{3}
$$

where

$$
\begin{aligned}
& \prod_{1}=\prod_{p \mid \ell_{1}} \sum_{\alpha \geq 0} \overline{\lambda_{f}\left(p^{\alpha+1}\right)} \lambda_{f}\left(p^{\alpha}\right) p^{-\alpha s} \\
& \prod_{2}=\prod_{p \mid \ell_{2}} \sum_{\alpha \geq 0} \lambda_{f}\left(p^{\alpha+1}\right) \overline{\lambda_{f}\left(p^{\alpha}\right)} p^{-\alpha s} \\
& \sum_{3}=\sum_{\left(n, \ell_{1} \ell_{2}\right)=1}\left|\lambda_{f}(n)\right|^{2} n^{-s} .
\end{aligned}
$$

For $p \mid \ell_{1}$, we have $\left(\psi_{M} \chi^{*}\right)(p)=\chi^{*}(p)$ and thus by (2.2) with $M N^{*}$ in place of $N$,

$$
\lambda_{f}\left(p^{\alpha}\right) \lambda_{f}(p)=\lambda_{f}\left(p^{\alpha+1}\right)+\chi^{*}(p) \lambda_{f}\left(p^{\alpha-1}\right) .
$$

After a little calculation with

$$
\overline{\chi^{*}(p) \lambda_{f}\left(p^{\alpha-1}\right)} \lambda_{f}\left(p^{\alpha}\right)=\overline{\chi^{*}(p)^{\alpha}} \lambda_{f}\left(p^{\alpha-1}\right) \lambda_{f}\left(p^{\alpha}\right)=\lambda_{f}\left(p^{\alpha-1}\right) \overline{\lambda_{f}\left(p^{\alpha}\right)}
$$

we see that

$$
\prod_{1}=\left(1+p^{-s}\right)^{-1} \overline{\lambda_{f}(p)} \sum_{\alpha \geq 0}\left|\lambda_{f}\left(p^{\alpha}\right)\right|^{2} p^{-\alpha s}
$$

whence by (2.19) and (2.18), we have

$$
\left(l_{1} l_{2}\right)^{k / 2} F(s)=\frac{\Gamma(s+k-1)}{(4 \pi)^{s+k-1}\left(l_{1}, l_{2}\right)^{s-1}} \frac{\overline{\lambda_{f}\left(\ell_{1}\right)} \ell_{1}^{1 / 2-s}}{\prod_{p \mid \ell_{1}}\left(1+p^{-s}\right)} \frac{\lambda_{f}\left(\ell_{2}\right) \ell_{2}^{1 / 2-s}}{\prod_{p \mid \ell_{2}}\left(1+p^{-s}\right)} L(s, f \otimes \bar{f})
$$

with

$$
L(s, f \otimes \bar{f})=\sum_{n \geq 1}\left|\lambda_{f}(n)\right|^{2} n^{-s} .
$$

We conclude our lemma from the residue of $F(s)$ at $s=1$. 
For $L M N^{*}=N$ and $f \in \mathrm{H}_{k}^{*}\left(M, \chi^{*}\right)$, we define

$$
\rho_{f}(L)=\sum_{b \mid L} \mu(b) b\left(\frac{\left|\lambda_{f}(b)\right|}{\nu(b)}\right)^{2}
$$

By (2.3), one can show that

$$
\rho_{f}(L)^{-1}=\frac{\nu(L)}{L} \sum_{l \mid L^{\infty}} \frac{\lambda_{f}\left(l^{2}\right) \overline{\chi^{*}(l)}}{l} .
$$

For $d \mid L$ and $f \in \mathrm{H}_{k}^{*}\left(M, \chi^{*}\right)$, we introduce

$$
f_{d}=\rho_{f}(d)^{-1 / 2} \sum_{c l=d} \mu(c) \frac{\overline{\lambda_{f}(c)} \sqrt{c}}{\nu(c)} f_{\mid l} .
$$

Then these $f_{d}$ 's altogether yield an orthogonal basis for $S_{k}(N, \chi)$, indeed we have

Lemma 2.2. Under the same assumption as in Lemma 2.1 we have for $d_{1}, d_{2} \mid L,\left(f_{d_{1}}, f_{d_{2}}\right)_{N^{\prime}}=$ $\delta_{d_{1}, d_{2}}(f, f)_{N^{\prime}}$ where $\delta_{,,}$. denotes the Kronecker delta.

Along the argument in [12], we unravel the construction of $f_{d}$ to explain the formula in (2.22) and to derive the lemma. To complete an orthogonal basis for $S_{k}(N, \chi)$, it remains to get an orthogonal basis for

$$
\operatorname{Span}_{\mathbb{C}}\left\{f_{\mid l}: l \mid L\right\},
$$

with $f \in \mathrm{H}_{k}^{*}\left(M, \chi^{*}\right)$. We consider the linear combination

$$
f_{d}=\sum_{l \mid L} x_{d}(l) f_{\mid l}
$$

where $x_{d}(\cdot)$ is supported on squarefree integers, and evaluate

$$
\delta_{f}\left(d_{1}, d_{2}\right)=\frac{\left(f_{d_{1}}, f_{d_{2}}\right)_{N^{\prime}}}{(f, f)_{N^{\prime}}}
$$

Now, by Lemma 2.1,

$$
\begin{aligned}
\delta_{f}\left(d_{1}, d_{2}\right)= & \sum_{l_{1} \mid L} \sum_{l_{2} \mid L} x_{d_{1}}\left(l_{1}\right) \overline{x_{d_{2}}\left(l_{2}\right)} \frac{\left(f_{\mid l_{1}}, f_{\mid l_{2}}\right)_{N^{\prime}}}{(f, f)_{N^{\prime}}} \\
= & \sum_{a \mid L} \sum_{\substack{\ell_{1}\left|L, \ell_{2}\right| L \\
\left(\ell_{1}, \ell_{2}\right)=1}} x_{d_{1}}\left(a \ell_{1}\right) \overline{x_{d_{2}}\left(a \ell_{2}\right)} \frac{\overline{\lambda_{f}\left(\ell_{1}\right)}}{\nu\left(\ell_{\ell_{1}}\right.} \frac{\lambda_{f}\left(\ell_{2}\right) \sqrt{\ell_{2}}}{\nu\left(\ell_{2}\right)} \\
= & \sum_{a \mid L} \sum_{b \mid L} \mu(b) b\left(\frac{\left|\lambda_{f}(b)\right|}{\nu(b)}\right)^{2} \\
& \times \sum_{\ell_{1}\left|L, \ell_{2}\right| L} x_{d_{1}}\left(a b \ell_{1}\right) \overline{x_{d_{2}}\left(a b \ell_{2}\right)} \frac{\overline{\lambda_{f}\left(\ell_{1}\right)} \sqrt{\ell_{1}}}{\nu\left(\ell_{1}\right)} \frac{\lambda_{f}\left(\ell_{2}\right) \sqrt{\ell_{2}}}{\nu\left(\ell_{2}\right)} \\
= & \sum_{c \mid L} \rho_{f}(c) y_{d_{1}}(c) \overline{y_{d_{2}}(c)},
\end{aligned}
$$


Omega result for supremum norms of Hecke-eigenforms

where $\rho_{f}(c)$ is defined as in $(2.20)$, and

$$
y_{d}(c)=\sum_{\ell \mid L} x_{d}(c \ell) \frac{\overline{\lambda_{f}(\ell)} \sqrt{\ell}}{\nu(\ell)} .
$$

(Note that $y_{d}$ is supported on squarefree integers.) If $f$ and $g$ are arithmetical functions supported on squarefree integers, then we have by moebius inversion formula (for $\mu$ ),

$$
g(c)=\sum_{\ell \mid L} f(c \ell), \forall c \in \mathbb{N} \quad \Leftrightarrow \quad f(l)=\sum_{c \mid L} g(c l) \mu(c), \forall l \in \mathbb{N} .
$$

Hence we deduce that

$$
x_{d}(l)=\sum_{c \mid L} \mu(c) y_{d}(c l) \frac{\overline{\lambda_{f}(c)} \sqrt{c}}{\nu(c)}
$$

Our goal is, by $(2.23)$, to make $\left(\delta_{f}\left(d_{1}, d_{2}\right)\right)$ into the identity matrix, so a natural choice is, from (2.24),

$$
y_{d}(c)=\rho_{f}(c)^{-1 / 2} \quad \text { if } c=d \text {, or } \quad y_{d}(c)=0 \quad \text { otherwise. }
$$

Thus if $l \mid d$, then by $(2.25)$,

$$
x_{d}(l)=\mu(c) \rho_{f}(d)^{-1 / 2} \frac{\overline{\lambda_{f}(c)} \sqrt{c}}{\nu(c)}
$$

where $c=d / l$. Otherwise, $x_{d}(l)=0$. This yields Lemma 2.2.

In virtue of the decomposition (2.15), we get an orthogonal basis

$$
\mathcal{B}_{k}(N, \chi)=\bigcup_{L M=N^{\prime}} \bigcup_{f \in \mathrm{H}_{k}^{*}\left(M, \chi^{*}\right)}\left\{f_{d}: \mathrm{d} \mid L\right\}
$$

for $S_{k}(N, \chi)$. The $n$th coefficient of $f_{d}$ is given by

$$
a_{f}(n)=\left(\frac{d}{\rho_{f}(d)}\right)^{1 / 2} n^{(k-1) / 2} \sum_{\substack{c l=d \\ l \mid n}} \mu(c) \frac{\overline{\lambda_{f}(c)}}{\nu(c)} \lambda_{f}\left(\frac{n}{l}\right) .
$$

So we have

$$
\sum_{f \in \mathcal{B}_{k}(N, \chi)}\|f\|^{-2} \frac{a_{f}(m) \overline{a_{f}(n)}}{(m n)^{(k-1) / 2}}=\sum_{L M=N^{\prime}} \sum_{f \in \mathrm{H}_{k}^{*}\left(M, \chi^{*}\right)}\|f\|^{-2} \Lambda_{L, f}(m, n)
$$

where

$$
\Lambda_{L, f}(m, n)=\sum_{d \mid L} \frac{d}{\rho_{f}(d)} \sum_{\substack{c l=d \\ l \mid m}} \mu(c) \frac{\overline{\lambda_{f}(c)}}{\nu(c)} \lambda_{f}\left(\frac{m}{l}\right) \sum_{\substack{e t=d \\ t \mid n}} \mu(e) \frac{\lambda_{f}(e)}{\nu(e)} \overline{\lambda_{f}\left(\frac{n}{t}\right)}
$$

and $\|f\|^{2}=(f, f)_{N^{\prime}}=\left(f_{d}, f_{d}\right)_{N^{\prime}}$ by Lemma 2.2. Assume $\left(m, n, N^{\prime}\right)=1$, then $(l, t)=1$ and $d$ is of the form $b t l$ in the last two sums. It follows that

$$
=\sum_{b \mid L} \frac{b}{\rho_{f}(b)}\left(\frac{\left|\lambda_{f}(b)\right|}{\nu(b)}\right)^{2} \sum_{\delta \mid L / b} \frac{\delta \mu(\delta)}{\rho_{f}(\delta) \nu(\delta)} \sum_{\substack{l t=\delta \\ l|m, t| n}} \lambda_{f}(l) \overline{\lambda_{f}(t)} \lambda_{f}\left(\frac{m}{l}\right) \overline{\lambda_{f}\left(\frac{n}{t}\right)} .
$$


Note that

$$
\lambda_{f}(l) \lambda_{f}(m / l)=\sum_{v \mid(l, m / l)}\left(\psi_{M} \chi^{*}\right)(v) \lambda_{f}\left(\frac{m}{v^{2}}\right)
$$

which is simply $\lambda_{f}(m)$ if $(l, m / l)=1$. We assume $\left(m, N^{\prime 2}\right) \mid N^{\prime}$, thus $(l, m / l)=1$ for $l \mid N^{\prime}$. Under the assumption that $\left(m, n, N^{\prime}\right)=1$ and $\left(m n, N^{\prime 2}\right) \mid N^{\prime}$, we get that

$$
\Lambda_{L, f}(m, n)=\lambda_{f}(m) \overline{\lambda_{f}(n)} \sum_{b \mid L} \frac{b}{\rho_{f}(b)}\left(\frac{\left|\lambda_{f}(b)\right|}{\nu(b)}\right)^{2} \sum_{\delta \mid L / b} \frac{\delta \mu(\delta)}{\rho_{f}(\delta) \nu(\delta)} \sum_{\substack{l t=\delta \\ l|m, t| n}} 1 .
$$

As $((m, L),(n, L))=1$, the multiple sum over $b, \delta, l, t$ in the last line equals

$$
\begin{aligned}
& \prod_{p \mid(m, L)}\left(1-\frac{p}{\rho_{f}(p) \nu(p)}+\frac{p}{\rho_{f}(p)}\left(\frac{\left|\lambda_{f}(p)\right|}{\nu(p)}\right)^{2}\right) \\
& \times \prod_{p \mid(n, L)}\left(1-\frac{p}{\rho_{f}(p) \nu(p)}+\frac{p}{\rho_{f}(p)}\left(\frac{\left|\lambda_{f}(p)\right|}{\nu(p)}\right)^{2}\right) \\
& \times \prod_{p \mid L /(m n, L)}\left(1+\frac{p}{\rho_{f}(p)}\left(\frac{\left|\lambda_{f}(p)\right|}{\nu(p)}\right)^{2}\right),
\end{aligned}
$$

which can be simplified into

$$
\rho_{f}(L)^{-1} \prod_{p \mid(m n, L)}(p+1)^{-1}
$$

as

$$
\frac{p}{\rho_{f}(p)}\left(\frac{\left|\lambda_{f}(p)\right|}{\nu(p)}\right)^{2}=\rho_{f}(p)^{-1}-1
$$

by (2.20). In summary, for $\left(m, n, N^{\prime}\right)=1$ and $\left(n, N^{\prime 2}\right) \mid N^{\prime}$ we have from (2.26) and (2.27),

$$
\begin{aligned}
\Delta_{N}(m, n):= & \frac{\Gamma(k-1)}{(4 \pi)^{k-1}} \sum_{f \in \mathcal{B}_{k}(N, \chi)}\|f\|^{-2} \frac{a_{f}(m) \overline{a_{f}(n)}}{(m n)^{(k-1) / 2}} \\
= & \frac{\Gamma(k-1)}{(4 \pi)^{k-1}} \sum_{L M=N^{\prime}} \nu((m n, L))^{-1} \rho_{f}(L)^{-1} \\
& \times \sum_{f \in \mathrm{H}_{k}^{*}\left(M, \chi^{*}\right)}\|f\|^{-2} \lambda_{f}(m) \overline{\lambda_{f}(n)} .
\end{aligned}
$$

Now we are ready to express the sum over primitive forms in terms of $\Delta_{N}(m, n)$.

Suppose $\left(m, N^{\prime}\right)=1$. Then $\nu((m n, L))=\nu(n, L)$ and $\|f\|^{2}=\|f\|_{N^{\prime}}^{2}=\nu(L)\|f\|_{M}^{2}$ if $L M N^{*}=N$, see (2.16) and (2.17). By (2.21), we deduce that

$$
\Delta_{N}(m, n)=\sum_{L M=N^{\prime}} \frac{1}{L \nu((n, L))} \sum_{l \mid L^{\infty}} \frac{\overline{\chi^{*}(l)}}{l} \Delta_{M N^{*}}^{*}\left(m l^{2}, n\right)
$$

where

$$
\Delta_{M N^{*}}^{*}(m, n)=\frac{\Gamma(k-1)}{(4 \pi)^{k-1}} \sum_{f \in \mathrm{H}_{k}^{*}\left(M, \chi^{*}\right)}\|f\|_{M}^{-2} \lambda_{f}(m) \overline{\lambda_{f}(n)} .
$$


Omega result for supremum norms of Hecke-eigenforms

This implies that for $\left(m, N^{\prime}\right)=1$ and $\left(n, N^{\prime 2}\right) \mid N^{\prime}$,

$$
\begin{aligned}
\Delta_{N}^{*}(m, n) & =\Delta_{N^{\prime} N^{*}}^{*}(m, n) \\
& =\sum_{L M=N^{\prime}} \frac{\mu(L)}{L \nu((n, L))} \sum_{l \mid L^{\infty}} \frac{\overline{\chi^{*}(l)}}{l} \Delta_{M N^{*}}\left(m l^{2}, n\right),
\end{aligned}
$$

by the following lemma.

Lemma 2.3. Let $g$ be a completely multiplicative function and $f$ be multiplicative. Suppose the functions $F$ and $G$ satisfies that for all squarefree $N$ and $(m, N)=1$,

$$
G(N, m)=\sum_{L M=N} f(L) \sum_{\ell \mid L^{\infty}} g(\ell) F\left(M, m \ell^{2}\right)
$$

Then we have

$$
F(N, m)=\sum_{L M=N} \mu(L) f(L) \sum_{\ell \mid L^{\infty}} g(\ell) G\left(M, m \ell^{2}\right)
$$

for squarefree $N$ and $(m, N)=1$.

Proof. This can be shown by direct verification. Assume $N$ be squarefree and $(m, N)=1$. We have

$$
\begin{aligned}
& \sum_{P R=N} \mu(P) f(P) \sum_{v \mid P^{\infty}} g(v) \sum_{L M=R} f(L) \sum_{\ell \mid L^{\infty}} g(\ell) F\left(M, m v^{2} \ell^{2}\right) \\
= & \sum_{Q M=N} f(Q) \sum_{q \mid Q^{\infty}} g(q) F\left(M, m q^{2}\right) \sum_{P \mid Q} \mu(P) \\
= & F(M, m),
\end{aligned}
$$

noting that $\left(m v^{2}, R\right)=1$ for $v \mid P^{\infty}$ and each $q$ decomposes uniquely into $q=v \ell$.

The Petersson trace formula states that

$$
\Delta_{N}(m, n)=\delta_{m, n}+2 \pi i^{k} \sum_{\substack{c \geq 1 \\ N \mid c}} c^{-1} S_{\chi}(m, n, c) J_{k-1}\left(\frac{4 \pi \sqrt{m n}}{c}\right)
$$

where $S_{\chi}(m, n, c)=\sum_{a d \equiv 1(c)} \chi(d) e((a m+d n) / c)$. Applying the Weil bound (in weak form)

$$
S_{\chi}(m, n, c) \ll c^{1 / 2+\varepsilon}(m, n, c)^{1 / 2}
$$

and the estimate

$$
J_{k-1}(x) \ll k^{-1 / 3} \min \left(1, \frac{x}{k}\right),
$$

we infer that the sum over $c$ is

$$
\ll \quad k^{-1 / 3} N^{\varepsilon-1 / 2}(m, n, N)^{1 / 2} \sum_{c \geq 1} c^{\varepsilon-1 / 2}(m, n, c)^{1 / 2} \min \left(1, \frac{\sqrt{m n}}{k N c}\right) .
$$

If $\sqrt{m n} / k N \leq 1$, then it is plainly

$$
\begin{aligned}
& \ll \frac{\sqrt{m n}(m, n, N)^{1 / 2}}{k^{4 / 3} N^{3 / 2-\varepsilon}} \sum_{c \geq 1} c^{\varepsilon-3 / 2}(m, n, c)^{1 / 2} \\
& \ll \frac{(m n)^{1 / 2+\varepsilon}}{k^{4 / 3} N^{3 / 2-\varepsilon}}(m, n, N)^{1 / 2} .
\end{aligned}
$$


Otherwise, it is

$$
\begin{aligned}
& \ll \quad k^{-1 / 3} N^{\varepsilon-1 / 2}(m, n, N)^{1 / 2} \sum_{1 \leq c \leq \sqrt{m n} /(k N)} c^{\varepsilon-1 / 2}(m, n, c)^{1 / 2} \\
& +\frac{\sqrt{m n}(m, n, N)^{1 / 2}}{k^{4 / 3} N^{3 / 2-\varepsilon}} \sum_{c \geq \sqrt{m n} /(k N)} c^{\varepsilon-3 / 2}(m, n, c)^{1 / 2} \\
& \ll \quad \frac{(m n)^{1 / 4}}{k^{5 / 6} N}(m, n, N)^{1 / 2}(k N m n)^{\varepsilon} .
\end{aligned}
$$

We thus conclude from (2.30) the asymptotic formula,

$$
\begin{aligned}
& \Delta_{N}(m, n) \\
= & \delta_{m, n}+O\left((k N m n)^{\varepsilon}(m, n, N)^{1 / 2} \frac{(m n)^{1 / 4}}{k^{5 / 6} N} \min \left(1, \frac{(m n)^{1 / 4}}{k^{1 / 2} N^{1 / 2}}\right)\right) .
\end{aligned}
$$

In view of (2.29), we evaluate

$$
\sum_{L M=N^{\prime}} \frac{\mu(L)}{L \nu((n, L))} \sum_{l \mid L^{\infty}} \frac{\overline{\chi^{*}(l)}}{l} \delta_{m l^{2}, n}
$$

and

$$
\begin{aligned}
& (k N m n)^{\varepsilon}\left(m, n, N^{*}\right)^{1 / 2} \frac{(m n)^{1 / 4}}{k^{5 / 6} N} \\
& \times \sum_{L M=N^{\prime}} \frac{1}{L \nu((n, L))} \sum_{l \mid L^{\infty}} l^{-1 / 2+\varepsilon} \min \left(1, \frac{(m n)^{1 / 4} l^{1 / 2}}{k^{1 / 2}\left(M N^{*}\right)^{1 / 2}}\right),
\end{aligned}
$$

where $\left(m, N^{\prime}\right)=1$ and $\left(n, N^{\prime 2}\right) \mid N^{\prime}$. Observing that $l=1$ and $(n, L)=1$ for a nonzero kronecker delta, we infer that (2.31) equals

$$
\sum_{L \mid N^{\prime}} \frac{\mu(L)}{L} \delta_{m, n}=\frac{\phi\left(N^{\prime}\right)}{N^{\prime}} \delta_{m, n}
$$

Denoting $Y=k^{1 / 2} N^{1 / 2} /(m n)^{1 / 4}$, we see that $(2.32)$ is

$$
\ll(k N m n)^{\varepsilon}\left(m, n, N^{*}\right)^{1 / 2} \frac{(m n)^{1 / 4}}{k^{5 / 6} N} \sum_{L M=N^{\prime}} \frac{1}{L} \sum_{l \mid L^{\infty}} l^{-1 / 2+\varepsilon} \min \left(1, l^{1 / 2} L^{1 / 2} / Y\right)
$$

Splitting accordingly, the double sum is

$$
\ll Y^{-1} \sum_{\substack{L M=N^{\prime} \\ L \leq Y^{2}}} \frac{1}{L^{1 / 2}} \sum_{\substack{l \mid L^{\infty} \\ l \leq Y^{2} / L}} l^{\varepsilon}+\sum_{\substack{L M=N^{\prime} \\ L \leq Y^{2}}} \frac{1}{L} \sum_{\substack{l \mid L^{\infty} \\ l>Y^{2} / L}} l^{-1 / 2+\varepsilon}+\sum_{\substack{L M=N^{\prime} \\ L>Y^{2}}} \frac{1}{L} \sum_{l \mid L^{\infty}} l^{-1 / 2+\varepsilon} .
$$

Note that

$$
\sum_{\substack{l \mid L^{\infty} \\ l \geq A}} l^{a} \ll A^{a+\varepsilon} \sum_{l \mid L^{\infty}} l^{-\varepsilon} \ll A^{a+\varepsilon} \varepsilon^{c \omega(L)}
$$

for some constant $c>0$, where $\omega(L)$ is the number of distinct prime factors of $L$. Separating into $Y \geq 1$ or not, we infer that $(2.32)$ is

$$
\ll \quad(k N m n)^{\varepsilon}\left(m, n, N^{*}\right)^{1 / 2} \frac{(m n)^{1 / 4}}{k^{5 / 6} N} \min \left(1, \frac{(m n)^{1 / 4}}{k^{1 / 2} N^{1 / 2}}\right) .
$$


Omega result for supremum norms of Hecke-eigenforms

This yields an asymptotic formula for (2.29): for $\left(m, N^{\prime}\right)=1$ and $\left(n, N^{\prime 2}\right) \mid N^{\prime}$,

$$
\begin{aligned}
& \Delta_{M N^{*}}^{*}(m, n) \\
= & \frac{\phi\left(N^{\prime}\right)}{N^{\prime}} \delta_{m, n}+O\left((k N m n)^{\varepsilon}\left(m, n, N^{*}\right)^{1 / 2} \frac{(m n)^{1 / 4}}{k^{5 / 6} N} \min \left(1, \frac{(m n)^{1 / 4}}{k^{1 / 2} N^{1 / 2}}\right)\right) .
\end{aligned}
$$

\section{Proof of Theorems}

Let $f \in \mathrm{H}_{k}^{*}\left(N^{\prime}, \chi^{*}\right)$ be a primitive form in $S_{k}(N, \chi)$ and admit the series $f(z)=\sum_{n \geq 1} \lambda_{f}(n) n^{(k-1) / 2} e(n z)$, normalized as in $\S 2$. By [7] and [9], its $L^{2}$-norm

$$
\|f\|^{2}=\int_{\Gamma_{0}(N) \backslash \mathbb{H}} y^{k}|f(z)|^{2} \frac{d x d y}{y^{2}}
$$

satisfies

$$
N(\log N)^{-1+o(1)} \ll_{k}\|f\|^{2} \ll_{k} N(\log N)^{1+o(1)} .
$$

This is well-known (see [11, Chapter 7] for instance) that

$$
\int_{0}^{\infty} f(i y) y^{k / 2} \frac{d y}{y}=(2 \pi)^{-k / 2} \Gamma(k / 2) L(1 / 2, f) .
$$

By Deligne's bound, we get that for $y \geq 1$,

$$
f(i y) \ll e^{-2 \pi y} \sum_{n \geq 1} n^{(k-1) / 2+\varepsilon} e^{-2 \pi n} \ll_{k} e^{-2 \pi y} .
$$

When $0 \leq y \leq N^{-1 / 2}$, we have by $(2.1)$,

$$
\eta_{f} f(i y)=i^{k} N^{-k / 2} y^{-k} \overline{f(i / N y)},
$$

and thus,

$$
\int_{0}^{1 / N} y^{k / 2} f(i y) \frac{d y}{y}=i^{k} \overline{\eta_{f}} \int_{1}^{\infty} y^{k / 2} \overline{f(i y)} \frac{d y}{y} .
$$

Therefore, by (3.2),

$$
\int_{1 / N}^{1} y^{k / 2} f(i y) \frac{d y}{y}=(2 \pi)^{-k / 2} \Gamma(k / 2) L(1 / 2, f)+O_{k}(1),
$$

whence it follows

$$
\sup _{1 / N \leq y \leq 1} y^{k / 2}|f(i y)| \gg_{k}(\log N)^{-1}(|L(1 / 2, f)|-O(1)) .
$$

This reduces the problem to pick $f$ with large $|L(1 / 2, f)|$.

To this end we apply the resonance method of Soundararajan. (Readers are referred to his paper [23] for the best exposition.) Define

$$
\Lambda(s, f)=\left(\frac{\sqrt{N}}{2 \pi}\right)^{s} \Gamma(s+(k-1) / 2) L(s, f)
$$


Then we have the functional equation (see [11, (7.17)])

$$
\Lambda(s, f)=i^{k} \overline{\eta_{f}} \overline{\Lambda(1-\bar{s}, f)} .
$$

Let $G(s)=\Gamma(A-s) \Gamma(A+s) / \Gamma(A)^{2}$ where $A>0$ is a large parameter up to our disposal. The standard argument using the residue theorem gives

$$
\begin{aligned}
& \left(\frac{\sqrt{N}}{2 \pi}\right)^{1 / 2} \Gamma(k / 2) L(1 / 2, f) \\
= & \frac{1}{2 \pi i} \int_{(2)} \Lambda(1 / 2+s, f) N^{s / 2} G(s) \frac{d s}{s}-\frac{i^{k} \overline{\eta_{f}}}{2 \pi i} \int_{(-2)} \overline{\Lambda(1 / 2-\bar{s}, f)} N^{s / 2} G(s) \frac{d s}{s} .
\end{aligned}
$$

After a change of variable $-\bar{s}$ into $s$, the second integral in the last line equals

$$
\overline{\int_{(2)} \Lambda(1 / 2+s, f) N^{-s / 2} G(s) \frac{d s}{s}} .
$$

Thus,

$$
\begin{aligned}
L(1 / 2, f) & =\sum_{n \geq 1} \frac{\lambda_{f}(n)}{\sqrt{n}} V\left(\frac{n}{N}\right)+i^{k} \overline{\eta_{f}} \sum_{n \geq 1} \frac{\overline{\lambda_{f}(n)}}{\sqrt{n}} V(n) \\
& =S_{1}(f)+S_{2}(f), \text { say, }
\end{aligned}
$$

where

$$
V(y)=\frac{\Gamma(k / 2)^{-1}}{2 \pi i} \int_{(2)} \Gamma(s+k / 2) G(s)(2 \pi y)^{-s} \frac{d s}{s}
$$

satisfies $\overline{V(y)}=V(y)$.

Clearly $V(y) \ll y^{-A}$ if $y \geq 1$. Thus, for any $\varepsilon>0$, the sum in $S_{1}(f)$ over $n \geq N^{1+\varepsilon}$ is negligible by setting $A=A(\varepsilon)$ to be a sufficiently large constant. Similarly we may neglect the terms with $n \geq N^{\varepsilon}$ in $S_{2}(f)$. We split $n$ into $n=n^{\prime} h^{2}$ where $\left(n^{\prime}, N^{\prime 2}\right) \mid N^{\prime}$ and $h \mid N^{\prime \infty}$, then $\lambda_{f}(n)=\lambda_{f}\left(n^{\prime}\right) \chi^{*}(h) / h$ by $(2.2)$. Hence,

$$
\begin{aligned}
S_{1}(f) & =\sum_{n \leq N^{1+\varepsilon}}^{\prime} \frac{\lambda_{f}(n)}{\sqrt{n}} \sum_{h \mid N^{\prime} \infty} \frac{\chi^{*}(h)}{h^{2}} V\left(\frac{n h^{2}}{N}\right)+O\left(N^{-1}\right) \\
& =\sum_{n \leq N^{1+\varepsilon}}^{\prime} \frac{\lambda_{f}(n)}{\sqrt{n}} V^{*}\left(\frac{n}{N}\right)+O\left(N^{-1}\right)
\end{aligned}
$$

where the summation $\sum^{\prime}$ runs over $n$ satisfying the condition $\left(n, N^{\prime 2}\right) \mid N^{\prime}$, and

$$
\begin{aligned}
V^{*}(y) & :=\sum_{h \mid N^{\prime \infty}} \frac{\chi^{*}(h)}{h^{2}} V\left(y h^{2}\right) \\
& =\frac{\Gamma(k / 2)^{-1}}{2 \pi i} \int_{(2)} \Gamma(s+k / 2) G(s) \prod_{p \mid N^{\prime}}\left(1-\chi^{*}(p) p^{-(2+2 s)}\right)^{-1}(2 \pi y)^{-s} \frac{d s}{s} \\
& =\prod_{p \mid N^{\prime}}\left(1-\chi^{*}(p) p^{-2}\right)^{-1}+O\left(y^{1 / 4} N^{\varepsilon}\right) .
\end{aligned}
$$


Omega result for supremum norms of Hecke-eigenforms

Here and in the sequel, we allow the implied constants to be dependent of $k$ and $\varepsilon$.

Let $X=N^{1 / 11}$ and $L=\frac{1}{2} \sqrt{(\log X)\left(\log _{2} X\right)}$. Define the multiplicative function $r(\cdot)$ supported on squarefree integers with

$$
|r(p)|= \begin{cases}L /(\sqrt{p} \log p) & \text { if } L^{2} \leq p \leq \exp \left((\log L)^{2}\right) \text { and } p \nmid N, \\ 0 & \text { otherwise. }\end{cases}
$$

The choice of $r(p)$ stems from a delicate optimization by Soundararajan, see [23, Theorem 1]. Introduce $\omega(f)=(4 \pi)^{1-k} \Gamma(k-1)\|f\|_{N^{\prime}}^{-2}$, and

$$
\begin{aligned}
R_{X}(f) & =\left|\sum_{a \leq X} r(a) \lambda_{f}(a)\right|^{2} \\
& =\sum_{a, b \leq X} r(a) \overline{r(b)} \chi(a) \overline{\sum_{d \mid(a, b)} \chi(d) \lambda_{f}\left(\frac{a b}{d^{2}}\right)},
\end{aligned}
$$

by (2.2). Firstly we recall the definition in (2.28). Squaring out $R_{X}(f)$, we infer by $(2.33)$ (and noting that $(a b, N)=1$ for nonvanishing $r(a) \overline{r(b)}$ from the definition of $r)$,

$$
\begin{aligned}
& \sum_{f \in \mathrm{H}_{k}^{*}\left(N^{\prime}, \chi^{*}\right)} \omega(f) R_{X}(f) \\
= & \sum_{a, b \leq X} r(a) \overline{r(b)} \Delta_{N}^{*}(a, b) \\
= & \sum_{a, b \leq X} r(a) \overline{r(b)}\left\{\frac{\phi\left(N^{\prime}\right)}{N^{\prime}} \delta_{a, b}+O\left(N^{\varepsilon-1 / 2}(a b)^{1 / 4+\varepsilon}\right)\right\} \\
= & \frac{\phi\left(N^{\prime}\right)}{N^{\prime}} \sum_{a \leq X}|r(a)|^{2}+O\left(N^{\varepsilon-1 / 2} X^{3 / 2}\left(\sum_{a \leq X} a^{-1 / 2}|r(a)|\right)^{2}\right) .
\end{aligned}
$$

We apply Rankin's trick to deal with the first summand. Generally suppose $f$ is a nonnegative multiplicative function supported on squarefree integers and $\sum_{p} f(p)^{2}\left(p^{\alpha}-1\right)^{2} \ll 1$. Then we have for any $d$ and $Y$,

$$
\begin{aligned}
\sum_{\substack{a \leq Y \\
(a, d)=1}} f(a) & =\prod_{p \nmid d}(1+f(p))+O\left(Y^{-\alpha} \prod_{p \nmid d}\left(1+f(p) p^{\alpha}\right)\right. \\
& =\prod_{p \nmid d}(1+f(p))\left(1+O\left(Y^{-\alpha} \exp \left(\sum_{p} f(p)\left(p^{\alpha}-1\right)\right)\right)\right) .
\end{aligned}
$$

Taking $Y=X, f(p)=|r(p)|^{2}, d=1$ and $\alpha=1 /(\log L)^{3}$ (so that $p^{\alpha}-1 \leq 2 \alpha \log p$ for $\left.L^{2} \leq p \leq \exp \left((\log L)^{2}\right)\right)$, then

$$
\begin{aligned}
\sum_{p}|r(p)|^{2}\left(p^{\alpha}-1\right) & \leq 2 \alpha L^{2} \sum_{L^{2} \leq p}(p \log p)^{-1} \\
& \leq \alpha L^{2} \frac{(1+o(1))}{\log L} \\
& \leq \frac{1+o(1)}{2} \alpha \log X
\end{aligned}
$$


We deduce that

$$
\sum_{a \leq X}|r(a)|^{2}=\prod_{p}\left(1+r(p)^{2}\right)\left\{1+O\left(X^{-\alpha / 3}\right)\right\}
$$

As above,

$$
\left.\sum_{p} p^{-1 / 2}|r(p)|\left(p^{\alpha}-1\right)\right) \ll \alpha L \log _{2} L
$$

and

$$
\sum_{p} p^{-1 / 2}|r(p)| \ll L(\log L)^{-1} .
$$

We have from (3.9) the crude bound,

$$
\sum_{a \leq X} a^{-1 / 2}|r(a)| \ll e^{L} \ll N^{\varepsilon} .
$$

It follows from (3.8) that

$$
\begin{aligned}
\sum_{f \in \mathrm{H}_{k}^{*}\left(N^{\prime}, \chi^{*}\right)} \omega(f) R_{X}(f)= & \frac{\phi\left(N^{\prime}\right)}{N^{\prime}} \prod_{p}\left(1+r(p)^{2}\right)\left\{1+O\left(X^{-\alpha / 3}\right)\right\} \\
& +O\left(N^{\varepsilon-1 / 2} X^{3 / 2}\right)
\end{aligned}
$$

and is thus $\ll N^{\varepsilon}$.

Next from (3.5) and (3.7), it follows that

$$
\begin{aligned}
& \sum_{f \in \mathrm{H}_{k}^{*}\left(N^{\prime}, \chi^{*}\right)} \omega(f) R_{X}(f) S_{1}(f) \\
= & \sum_{n \leq N^{1+\varepsilon}} \frac{1}{\sqrt{n}} V^{*}\left(\frac{n}{N}\right) \sum_{a, b \leq X} r(a) \overline{r(b)} \chi(a) \sum_{d \mid(a, b)} \overline{\chi(d)} \\
& \times \sum_{f \in \mathrm{H}_{k}^{*}\left(N^{\prime}, \chi^{*}\right)} \omega(f) \lambda_{f}(n) \overline{\lambda_{f}\left(\frac{a b}{d^{2}}\right)} \\
+ & O\left(N^{-1} \sum_{f \in \mathrm{H}_{k}^{*}\left(N^{\prime}, \chi^{*}\right)} \omega(f) R_{X}(f)\right) .
\end{aligned}
$$

By the estimate for (3.14), the $O$-term in (3.15) is clearly $\ll N^{\varepsilon-1}$.

Applying (2.33) to $\Delta_{N}^{*}\left(a b / d^{2}, n\right)$, the multiple sum in (3.15) is equal to

$$
\begin{aligned}
& \frac{\phi\left(N^{\prime}\right)}{N^{\prime}} \sum_{a, b \leq X} \chi(a) \frac{r(a) \overline{r(b)}}{\sqrt{a b}} \sum_{d \mid(a, b)} \overline{\chi(d)} d V^{*}\left(\frac{a b}{d^{2} N}\right) \\
& +O\left(N^{\varepsilon-1} \sum_{a, b \leq X}|r(a) r(b)|(a b)^{1 / 4} \sum_{n \leq N^{1+\varepsilon}}^{\prime} n^{-1 / 4}\left(a b, n, N^{*}\right)^{1 / 2}\right) .
\end{aligned}
$$

Thus the $O$-term is $\ll N^{\varepsilon-1 / 4} X^{5 / 2}\left(\sum_{a \leq X} a^{-1 / 2}|r(a)|\right)^{2} \ll N^{\varepsilon-1 / 4} X^{5 / 2}$. 
Omega result for supremum norms of Hecke-eigenforms

Together with (3.6), we express (3.15) as

$$
\begin{aligned}
& \sum_{f \in \mathrm{H}_{k}^{*}\left(N^{\prime}, \chi^{*}\right)} \omega(f) R_{X}(f) S_{1}(f) \\
= & \frac{\phi\left(N^{\prime}\right)}{N^{\prime}} \prod_{p \mid N^{\prime}}\left(1-\chi^{*}(p) p^{-2}\right)^{-1} \sum_{a, b \leq X} \chi(a) \frac{r(a) r(b)}{\sqrt{a b}} \sum_{d \mid(a, b)} \overline{\chi(d)} d \\
+ & O\left(N^{\varepsilon-1 / 4} X\left(\sum_{a \leq X} a^{-1 / 2}|r(a)|\right)^{2}\right)+O\left(N^{\varepsilon-1 / 4} X^{5 / 2}\right),
\end{aligned}
$$

where the first $O$-term comes from the $O$-term in (3.6). It is $\ll N^{\varepsilon-1 / 4} X$, so absorbed by the last term. The ultimate task is to deal with the sum

$$
\begin{aligned}
& \sum_{a, b \leq X} \chi(a) \frac{r(a) \overline{r(b)}}{\sqrt{a b}} \sum_{d \mid(a, b)} \overline{\chi(d)} d \\
= & \sum_{d}|r(d)|^{2} \sum_{\substack{a \leq X / d \\
(a, d)=1}} \frac{r(a) \chi(a)}{\sqrt{a}} \sum_{\substack{b \leq X / d \\
(b, d)=1}} \frac{\overline{r(b)}}{\sqrt{b}} \\
= & \sum_{d \leq \sqrt{X}}+\sum_{d>\sqrt{X}}, \text { say, }
\end{aligned}
$$

where the empty sum means zero. The tail section $\sum_{d>\sqrt{X}}$ over $d \geq \sqrt{X}$ is plainly

$$
\begin{aligned}
& \ll X^{-\alpha / 2} \sum_{d} d^{\alpha}|r(d)|^{2}\left(\sum_{(a, d)=1} a^{-1 / 2}|r(a)|\right)^{2} \\
& \ll X^{-\alpha / 2} \sum_{d} d^{\alpha}|r(d)|^{2} \prod_{p \nmid d}\left(1+p^{-1 / 2}|r(p)|\right)^{2} \\
& \ll X^{-\alpha / 2} \prod_{p}\left(\left(1+p^{-1 / 2}|r(p)|\right)^{2}+p^{\alpha}|r(p)|^{2}\right),
\end{aligned}
$$

which equals

$$
\begin{aligned}
& \prod_{p}\left(\left(1+p^{-1 / 2} r(p) \chi(p)\right)\left(1+p^{-1 / 2} \overline{r(p)}\right)+|r(p)|^{2}\right) \\
& \times O\left(X^{-\alpha / 2} \exp \left(4 \sum_{p}|r(p)| p^{-1 / 2}+\sum_{p}|r(p)|^{2}\left(p^{\alpha}-1\right)\right)\right) .
\end{aligned}
$$

By (3.10) and (3.12), the above $O$-term is absorbed into $O\left(X^{-\alpha / 3}\right)$, so

$$
\sum_{d>\sqrt{X}}=O\left(X^{-\alpha / 3}\right) \prod_{p}\left(\left(1+p^{-1 / 2} r(p) \chi(p)\right)\left(1+p^{-1 / 2} \overline{r(p)}\right)+|r(p)|^{2}\right) .
$$

The main contribution arises from

$$
\sum_{d \leq \sqrt{X}}|r(d)|^{2} \sum_{\substack{a \leq X / d \\(a, d)=1}} \frac{r(a) \chi(a)}{\sqrt{a}} \sum_{\substack{b \leq X / d \\(b, d)=1}} \frac{\overline{r(b)}}{\sqrt{b}} .
$$

For $d \leq \sqrt{X}$, the same argument as in (3.9) with (3.11) gives

$$
\begin{aligned}
& \sum_{\substack{a \leq X / d \\
(a, d)=1}} a^{-1 / 2} r(a) \chi(a) \\
= & \prod_{p \nmid d}\left(1+p^{-1 / 2} r(p) \chi(p)\right)\left(1+O\left(X^{-\alpha / 3}\right)\right)
\end{aligned}
$$


and an analogous estimate for the sum over $b$. Thus, we obtain

$$
\left(1+O\left(X^{-\alpha / 3}\right)\right) \sum_{d \leq \sqrt{X}}|r(d)|^{2} \prod_{p \nmid d}\left(1+p^{-1 / 2} r(p) \chi(p)\right) \prod_{p \nmid d}\left(1+p^{-1 / 2} \overline{r(p)}\right)
$$

and with the Rankin trick again, the sum over $d \leq \sqrt{X}$ is

$$
\begin{aligned}
& \sum_{d \leq \sqrt{X}} \\
= & \left(1+O\left(X^{-\alpha / 3}\right)\right) \prod_{p}\left(\left(1+p^{-1 / 2} r(p) \chi(p)\right)\left(1+p^{-1 / 2} \overline{r(p)}\right)+|r(p)|^{2}\right) .
\end{aligned}
$$

Together with (3.19), we conclude that (3.18) equals

$$
\left(1+O\left(X^{-\alpha / 3}\right)\right) \prod_{p}\left(\left(1+p^{-1 / 2} r(p) \chi(p)\right)\left(1+p^{-1 / 2} \overline{r(p)}\right)+|r(p)|^{2}\right),
$$

and with (3.17),

$$
\begin{aligned}
& \sum_{f \in \mathrm{H}_{k}^{*}\left(N^{\prime}, \chi^{*}\right)} \omega(f) R_{X}(f) S_{1}(f) \\
= & \frac{\phi\left(N^{\prime}\right)}{N^{\prime}}\left(1+O\left(X^{-\alpha / 3}\right)\right) \prod_{p}\left(\left(1+p^{-1 / 2} r(p) \chi(p)\right)\left(1+p^{-1 / 2} \overline{r(p)}\right)+|r(p)|^{2}\right) \\
& +O\left(N^{\varepsilon-1} X^{5 / 2}\right) .
\end{aligned}
$$

By (2.2) and (2.4), $\overline{\eta_{f}}=\kappa \overline{\lambda_{f}(N)}$ where $\kappa=\sqrt{N^{\prime}} \overline{\tau(\bar{\chi})} / \sqrt{N^{*}}$ is indepedent of $f$ and has absolute value $\sqrt{N^{\prime}}$. The contribution of

$$
\sum_{f \in \mathrm{H}_{k}^{*}\left(N^{\prime}, \chi^{*}\right)} \omega(f) R_{X}(f) S_{2}(f)
$$

is negligible. Since

$$
\sum_{n \leq N^{\varepsilon}} n^{-1 / 2} V(n) \overline{\lambda_{f}(n) \lambda_{f}(N)}=\sum_{\substack{n h \leq N^{\varepsilon} \\(n, N)=1, h \mid N^{\infty}}} \frac{\overline{\lambda_{f}(n)}}{n^{1 / 2}} \frac{\overline{\lambda_{f}(h N)}}{h^{1 / 2}} V(n h),
$$

(3.21) is equal to

$$
\begin{aligned}
& \kappa \sum_{\substack{n h \leq N^{\varepsilon} \\
(n, N)=1, h \mid N^{\infty}}}(n h)^{-1 / 2} V(n h) \sum_{a, b \leq X} r(a) \overline{r(b)} \\
& \times \sum_{f \in \mathrm{H}_{k}^{*}\left(N^{\prime}, \chi^{*}\right)} \omega(f) \lambda_{f}(a) \overline{\lambda_{f}(b) \lambda_{f}(n) \lambda_{f}(h N)} \\
& +O\left(N^{-1} \sum_{f \in \mathrm{H}_{k}^{*}\left(N^{\prime}, \chi^{*}\right)} \omega(f) R_{X}(f)\right) .
\end{aligned}
$$

Removing the even power of $p \mid N^{\prime}$ in $h N$ by (2.2), we can apply the formula (2.33) to deduce

$$
\begin{aligned}
(3.21) & \ll N^{\prime 1 / 2} N^{* 1 / 2} \sum_{a, b \leq X}|r(a) r(b)| \sum_{n \leq N^{\varepsilon}} n^{-1 / 2}(a b n N)^{1 / 4} N^{\varepsilon-1} \\
& \ll X^{3 / 2} N^{\varepsilon-1 / 4}\left(\sum_{a \leq X} a^{-1 / 2}|r(a)|\right)^{2} \\
& \ll X^{3 / 2} N^{\varepsilon-1 / 4} .
\end{aligned}
$$


Omega result for supremum norms of Hecke-eigenforms

Altogether we infer with (3.4) and (3.20) that

$$
\begin{aligned}
& \sum_{f \in \mathrm{H}_{k}^{*}\left(N^{\prime}, \chi^{*}\right)} \omega(f) R_{X}(f) L(1 / 2, f) \\
= & \frac{\phi\left(N^{\prime}\right)}{N^{\prime}}\left(1+O\left(X^{-\alpha / 3}\right)\right) \prod_{p}\left(\left(1+p^{-1 / 2} r(p) \chi(p)\right)\left(1+p^{-1 / 2} r(p)\right)+r(p)^{2}\right) \\
& +O\left(X^{5 / 2} N^{\varepsilon-1 / 4}\right) .
\end{aligned}
$$

Note that

$$
\begin{aligned}
\sum_{p}|r(p)|^{2} & =L \sum_{L^{2} \leq p \leq \exp \left((\log L)^{2}\right)} \frac{1}{p(\log p)^{2}}+O\left(L \sum_{L^{2} \leq p \mid N} \frac{1}{p(\log p)^{2}}\right) \\
& =(1+o(1)) \frac{L}{(2 \log L)^{2}}+O\left(\frac{\log N}{L(\log L)^{3}}\right) \\
& =\left(\frac{1}{4}+o(1)\right) \frac{L}{(\log L)^{2}}
\end{aligned}
$$

for $L^{2} \gg \log N$ and

$$
\begin{aligned}
& \prod_{p}\left(\left(1+p^{-1 / 2} r(p) \chi(p)\right)\left(1+p^{-1 / 2} \overline{r(p)}\right)+|r(p)|^{2}\right) \\
= & \prod_{p}\left(1+|r(p)|^{2}\right) \times \exp \left(\sum_{p} p^{-1 / 2} \Re e(\overline{r(p)}+\chi(p) r(p))+O(1)\right) .
\end{aligned}
$$

By (3.14) and (3.22), we infer that

$$
\begin{aligned}
& \frac{\sum_{f \in \mathrm{H}_{k}^{*}\left(N^{\prime}, \chi^{*}\right)} \omega(f) R_{X}(f) L(1 / 2, f)}{\sum_{f \in \mathrm{H}_{k}^{*}\left(N^{\prime}, \chi^{*}\right)} \omega(f) R_{X}(f)} \\
= & \left(1+O\left(X^{-1 /\left(3(\log L)^{3}\right)}\right)\right) \exp \left(\sum_{p} p^{-1 / 2}(\overline{r(p)}+\chi(p) r(p))+O(1)\right) .
\end{aligned}
$$

We set $r(p)=|r(p)|$ for simplicity, then

$$
\begin{aligned}
& \sum_{p} p^{-1 / 2}(\overline{r(p)}+\chi(p) r(p)) \\
= & L \sum_{L^{2} \leq p \leq \exp \left((\log L)^{2}\right)} \frac{1}{p \log p} \\
& +O\left(L \sum_{L^{2} \leq p \mid N} \frac{1}{p \log p}+L \sum_{L^{2} \leq p \leq \exp \left((\log L)^{2}\right)} \frac{\chi^{*}(p)}{p \log p} \mid\right) \\
= & (1+o(1)) \frac{L}{2 \log L}+O\left(\frac{\log N}{L(\log L)^{2}}+L\left|\sum_{L^{2} \leq p \leq \exp \left((\log L)^{2}\right)} \frac{\chi^{*}(p)}{p \log p}\right|\right) .
\end{aligned}
$$

To control

$$
\sum_{L^{2} \leq p \leq \exp \left((\log L)^{2}\right)} \frac{\chi^{*}(p)}{p \log p}
$$


in the $O$-term, we invoke the following result (see [6, Chapter 20]): let

$$
\psi(x, \chi)=\sum_{n \leq x} \chi(n) \Lambda(n)
$$

where $\chi$ is a non-principal character $\bmod q$ and $\Lambda(\cdot)$ is the von-Mangoldt function, then for $q \leq \exp (c \sqrt{\log x})$

$$
\psi(x, \chi)=-\frac{x^{\beta}}{\beta}+O(x \exp (-c \sqrt{\log x}))
$$

where $c>0$ is an absolute constant and $\beta$ is the possible Siegel zero for $L(s, \chi)$. It is known that the Siegel zero does not exist for non-real character $\chi$ and satisfies, if exists,

$$
\beta \leq 1-C(\varepsilon) q^{-\varepsilon}
$$

for some ineffective constant $C(\varepsilon)>0$. Under the Grand Riemann Hypothesis (GRH), the much better estimate

$$
\psi(x, \chi) \ll \sqrt{x}(\log x)^{2}
$$

for $q \leq x$ (and non-principal $\chi$ ) will be available.

Using them, we obtain easily that

$$
\sum_{L^{2} \leq p \leq \exp \left((\log L)^{2}\right)} \frac{\chi^{*}(p)}{p \log p} \ll \frac{1}{(\log L)^{2}},
$$

if either

(i) $L(s, \chi)$ has no Siegel zero and $N^{*} \leq \exp (c \sqrt{\log L}) \leq \exp \left(c^{\prime} \sqrt{\log _{2} N}\right)$, or

(ii) $L(s, \chi)$ has a Siegel zero and $N^{*} \leq c(\varepsilon)(\log L)^{1 / \varepsilon} \leq c(\varepsilon)(\log L)^{1 / \varepsilon}$, or

(iii) GRH is true (and $\left.N^{*} \leq N\right)$.

Under the condition (i), (ii) or (iii), we can ensure the $O$-term in (3.24) to be $o(L / \log L)$, hence by $(3.23)$,

$$
\begin{aligned}
& \frac{\sum_{f \in \mathrm{H}_{k}^{*}\left(N^{\prime}, \chi^{*}\right)} \omega(f) R_{X}(f) L(1 / 2, f)}{\sum_{f \in \mathrm{H}_{k}^{*}\left(N^{\prime}, \chi^{*}\right)} \omega(f) R_{X}(f)} \\
= & \exp \left((1+o(1)) \frac{L}{2 \log L}\right) \\
= & \exp \left(\left(\frac{1}{2}+o(1)\right) \sqrt{\frac{\log N}{\log _{2} N}}\right) .
\end{aligned}
$$

This completes the proof of Theorem 2 with the positivity of $R_{X}(f)$, and by (3.3) and (3.1),

$$
\begin{aligned}
\sup _{1 / N \leq y \leq 1} y^{k / 2}|f(i y)| & \gg \exp \left(\left(\frac{1}{2}+o(1)\right) \sqrt{\frac{\log N}{\log _{2} N}}\right) \\
& \gg \exp \left(\left(\frac{1}{2}+o(1)\right) \sqrt{\frac{\log N}{\log _{2} N}}\right) N^{-1 / 2}\|f\|,
\end{aligned}
$$


Omega result for supremum norms of Hecke-eigenforms

which yields Theorem 1.

Acknowledgements The author wishes to thank the referees for the many valuable comments. He would also thank Dr. Jie Wu for his remarks and acknowledge the RGC grant (HKU $7032 / 06 \mathrm{P})$.

\section{References}

[1] A. Abbes \& E. Ullmo, Comparaison des mtriques d'Arakelov et de Poincaré sur $X_{0}(N)$, Duke Math. J. 80 (1995), pp.295-307.

[2] A. Atkin \& J. Lehner, Hecke operators on $\Gamma_{0}(m)$, Math. Ann. 185 (1970), pp.134-160.

[3] V. Blomer \& R. Holowinsky, Bounding sup-norms at cusp forms of large level, preprint.

[4] S. De Biévre, Quantum chaos: a brief first visit, Contemp. Math. 289, Amer. Math. Soc., Providence, RI, 2001.

[5] P. Deligne, La conjecture de Weil. I, Inst. Hautes tudes Sci. Publ. Math. 43 (1974), pp.273307.

[6] H. Davenport, Multiplicative number theory, 2nd edition, Springer-Verlag, 1980.

[7] J. Hoffstein \& P. Lockhart, Coefficients of Maass forms and the Siegel zero, Ann. Math. 140 (1994), pp.161-176.

[8] R. Holowinsky \& K. Soundararajan, Mass equidistribution for Hecke eigenforms, preprint, available at http://front.math.ucdavis.edu/ .

[9] D. Goldfeld, J. Hoffstein \& P. Lockhart, Appendix: An effective zero-free region, Ann. Math. 140 (1994), pp.177-181.

[10] H. Iwaniec, Small eigenvalues of Laplacian for $\Gamma_{0}(N)$, Acta Arith. 56 (1990), pp.65-82.

[11] H. Iwaniec, Topics in classical automorphic forms, AMS, 1997.

[12] H. Iwaniec, W. Luo \& P. Sarnak, Low lying zeros of families of $L$-functions, Inst. Hautes tudes Sci. Publ. Math. 91 (2000), 55-131 (2001).

[13] H. Iwaniec \& E. Kowalski, Analytic number theory, AMS, 2004.

[14] W.W. Li, Newforms and functional equations, Math. Ann. 212 (1975), pp.285-315.

[15] J. Jorgenson \& J. Kramer, Bounding the sup-norm of automorphic forms, Geom. Funct. Anal. 14 (2004), pp.1267-1277.

[16] J. Marklof, Arithmetic quantum chaos, Encyclopedia of Mathematical Physics, Oxford, 2006, Volume 1, pp.212-220. 
[17] P. Michel \& E. Ullmo, Points de petite hauteur sur les courbes modulaires $X_{0}(N)$, Invent. Math. 131 (1998), pp.645-674.

[18] A. Ogg, On the eigenvaules of Hecke operators, Math. Ann. 179 (1969), pp.101-108.

[19] Z. Rudnick \& P. Sarnak, The behaviour of eigenstates of arithmetic hyperbolic manifolds, Comm. Math. Phys. 161 (1994), pp.195-213.

[20] P. Sarnak, Arithmetic quantum chaos, Israel Math. Conf. Proc. 8, Bar-Ilan Univ., Ramat Gan, 1995, pp.183-236.

[21] P. Sarnak, Letter to Morawetz, available at http://www.math.princeton.edu/sarnak/.

[22] P. Sarnak, Recent Progress on QUE, available at http://www.math.princeton.edu/sarnak/.

[23] K. Soundararajan, Extreme values of zeta and $L$-functions, Math. Annalen, Math. Ann. 342 (2008), pp.467-486.

[24] K. Soundararajan, Quantum unique ergodicity for $S L_{2}(\mathbb{Z}) \backslash \mathbb{H}$, preprint at arXiv:0901.4060.

[25] H. Xia, On $L^{\infty}$ norms of holomorphic cusp forms, J. Number Theory 124 (2007), pp.325327. 